\title{
Association of ambulatory blood pressure monitoring with renal outcome in patients with chronic kidney disease
}

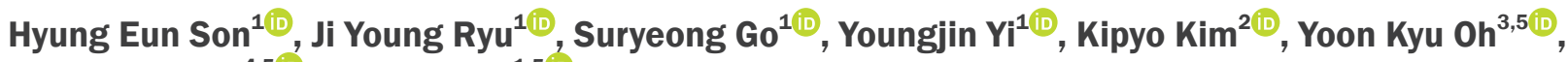 \\ Kook-Hwan $\mathrm{Oh}^{4,5}$, Ho Jun Chin ${ }^{1,5}$ \\ ${ }^{1}$ Department of Internal Medicine, Seoul National University Bundang Hospital, Seongnam, Republic of Korea \\ Department of Internal Medicine, Inha University Hospital, Incheon, Republic of Korea \\ ${ }^{3}$ Department of Internal Medicine, Seoul Metropolitan Government-Seoul National University Boramae Medical Center, Seoul, \\ Republic of Korea \\ ${ }^{4}$ Department of Internal Medicine, Seoul National University Hospital, Seoul, Republic of Korea \\ ${ }^{5}$ Department of Internal Medicine, Seoul National University College of Medicine, Seoul, Republic of Korea
}

\begin{abstract}
Background: The significance of ambulatory blood pressure (ABP) in Korean patients with chronic kidney disease $(C K D)$ in relation to renal outcome or death remains unclear. We investigated the role of ABP in predicting end-stage renal disease or death in patients with CKD.

Methods: We enrolled 387 patients with hypertension and CKD who underwent ABP monitoring and were followed for 1 year. Data on clinical parameters and outcomes from August 2014 to May 2018 were retrospectively collected. The composite endpoint was end-stage renal disease or death. Patients were grouped according to the mean ABP.

Results: There were 66 endpoint events, 52 end-stage renal disease cases, and 15 mortalities. Among all patients, one developed end-stage renal disease and died. Mean ABP in the systolic and diastolic phases were risk factors for the development of composite outcome with hazard ratios of 1.03 (95\% confidence interval $[\mathrm{Cl}], 1.01-1.04 ; P<$ $0.001)$ and $1.04(95 \% \mathrm{Cl}, 1.02-1.07 ; P=0.001)$ for every $1 \mathrm{mmHg}$ increase in $\mathrm{BP}$, respectively. Patients with mean ABP between $125 / 75$ and $130 / 80 \mathrm{mmHg}$ had a 2.56 -fold higher risk for the development of composite outcome $(95 \%$ $\mathrm{Cl}, 0.72-9.12 ; P=0.147$ ) as compared to those with mean $\mathrm{ABP} \leq 125 / 75 \mathrm{mmHg}$. Patients with mean $\mathrm{ABP} \geq 130 / 80$ $\mathrm{mmHg}$ had a 4.79 -fold higher risk (95\% $\mathrm{Cl}, 1.68-13.70 ; P=0.003)$ compared to those with mean $\mathrm{ABP} \leq 125 / 75$ $\mathrm{mmHg}$. Office blood pressure (OBP) was not a risk factor for the composite outcome when adjusted for covariates.

Conclusion: In contrast to OBP, ABP was a significant risk factor for end-stage renal disease or death in CKD patients.
\end{abstract}

Keywords: Blood pressure monitoring, ambulatory, Hypertension, Kidney failure, chronic

Received September 24, 2019; Revised November 23, 2019; Accepted December 3, 2019

Edited by Dong-Ryeol Ryu, Ewha Womans University, Seoul, Republic of Korea

Correspondence: Ho Jun Chin

Department of Internal Medicine, Seoul National University Bundang Hospital, 82 Gumi-ro 173beon-gil, Bundang-gu, Seongnam 13620, Republic of Korea. E-mail: mednep@hanmail.net

Copyright @ 2020 by The Korean Society of Nephrology

(a) This is an open-access article distributed under the terms of the Creative Commons Attribution Non-Commercial License (http://creativecommons.org/ licenses/by-nc-nd/4.0/), which permits unrestricted non-commercial use, distribution, and reproduction in any medium, provided the original work is properly cited. 


\section{Introduction}

According to the Korea National Health and Nutrition Examination Survey data, the overall prevalence of chronic kidney disease (CKD) in Korea is $8.2 \%$, and hypertension is an important factor in the development and progression of CKD [1]. Recently, the 2017 American College of Cardiology/American Heart Association (ACC/ AHA) guidelines suggested lowering the threshold of normal blood pressure (BP) to $<130 / 80 \mathrm{mmHg}$ in the general population and in patients with CKD [2].

Several guidelines have recommended different target $\mathrm{BP}$ values in patients with $\mathrm{CKD}$. Lowering the target $\mathrm{BP}$ to $<130 / 80 \mathrm{mmHg}$ has been recommended in patients with CKD [3]. Large cohort studies have demonstrated that an intensive office $\mathrm{BP}$ (ОВP) target has no benefit with respect to survival rate or renal outcomes [4-6]. However, the post-hoc study of the Systolic Blood Pressure Intervention Trial (SPRINT) study suggested that intensive BP treatment would improve the mortality rate in patients with CKD [7]. Furthermore, controversy remains about the appropriate $\mathrm{BP}$ target for improving the prognosis of patients with CKD.

Considering BP variations in a single patient with CKD, OBP might cause the misclassification of hypertension. In the report from the African American Study of Kidney disease trial, masked hypertension accounted for $70 \%$ of all well-controlled hypertension cases [8]. One study in 2013 showed that among 5,698 patients with CKD in Spain, the incidence of whitecoat hypertension was $28.8 \%$ and that of masked hypertension was $7 \%$ [9]. In previous studies, whitecoat and masked hypertension were reported as cardiovascular risk factors $[10,11]$. Therefore, ambulatory BP monitoring (ABPM) is useful considering the diverse BP patterns in patients with CKD.

The purpose of this study was to examine the significance of $\mathrm{ABP}$ in estimating clinical outcomes and to suggest an optimal BP target in patients with CKD.

\section{Methods}

\section{Patients and study design}

This study was an extension of a 12-month prospective cohort study that enrolled patients with hypertension and CKD stages 1 to 4 from August 2014 to May 2015 from
Seoul National University Bundang Hospital, Seoul Metropolitan Government-Seoul National University Boramae Medical Center, and Seoul National University Hospital [12]. The initial inclusion criteria were (1) age 20 to 75 years, (2) BP $\geq 140 / 90 \mathrm{mmHg}$, and/or (3) the use of the same BP medication since at least 2 weeks before enrollment. Patients with acute kidney injury, hospitalization, renal replacement therapy, previous kidney transplantation, uncontrolled arrhythmia, asthma, chronic obstructive pulmonary disease, and primary endocrine disorders except diabetes mellitus (DM) were excluded. Pregnant women and individuals who work night shifts were also excluded. OBP and 24-hour ABP were measured in all patients.

Clinical data and outcomes from August 2014 to May 2018 were retrospectively collected. The primary outcome was the incidence of end-stage renal disease (ESRD) or death (Fig. 1). ESRD was defined as the requirement of maintenance dialysis or kidney transplantation. Information about the development of ESRD was obtained from the electronic medical record (EMR) system of each hospital and ESRD registry of the Korea Society of Nephrology; information about death was collected from the EMR system of each hospital and database of the Ministry of

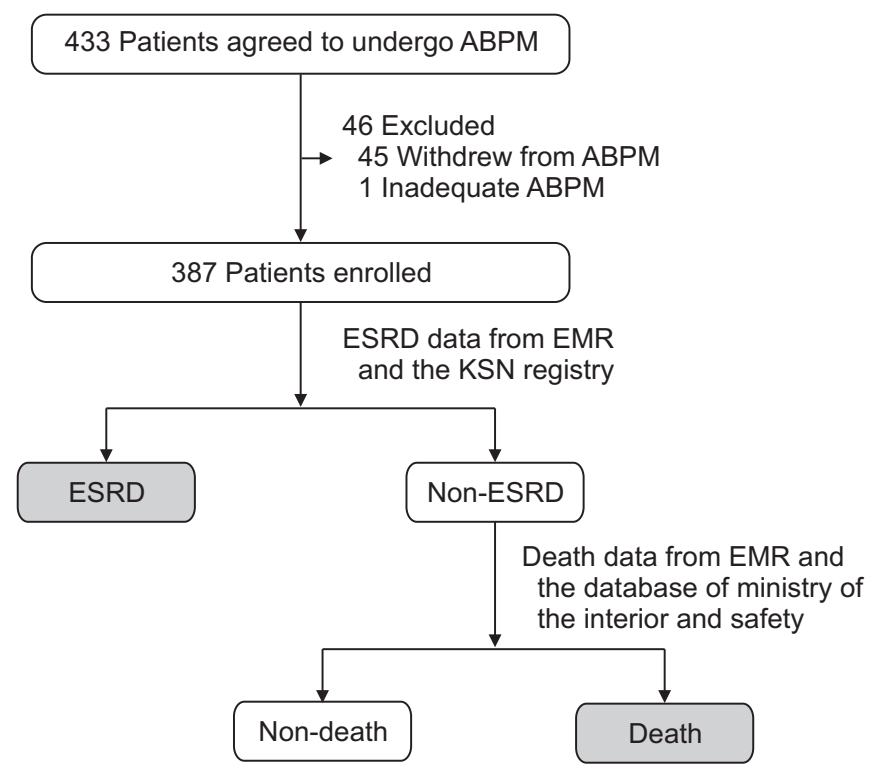

Figure 1. Diagram of the study population and collection of the composite outcome.

ABPM, ambulatory blood pressure monitoring; EMR, electronic medical record; ESRD, end-stage renal disease; KSN, Korean Society of Nephrology. 
the Interior and Safety by comparing the name and date of birth of each patient.

The present study protocol was reviewed and approved by the Institutional Review Board of Seoul National University Bundang Hospital (approval number: B-1809-490107), Seoul Metropolitan Government-Seoul National University Boramae Medical Center (approval number: 20181203/10-2018-107/011), and Seoul National University Hospital (approval number: 1812-030-991).

\section{Clinical and biochemical assessments}

Demographic information such as age, sex, medical history (hypertension, DM, and the use of antihypertensive drugs), blood tests (serum hemoglobin, albumin, blood urea nitrogen, creatinine, calcium, phosphorous, intact parathyroid hormone, total cholesterol, high-density lipoprotein cholesterol, low-density lipoprotein cholesterol, and triglycerides), and urine protein-creatinine ratio (UPCR) was obtained from the EMR system at the time of enrolment. Renal function was measured using serum isotope dilution mass spectrometry traceable creatinine, estimated glomerular filtration rate (eGFR) calculated using the CKD Epidemiology Collaboration (CKD-EPI) equation, and UPCR in random urine samples.

\section{Measurement of $A B P$}

OBP was measured by medical staff using a mercury sphygmomanometer with an appropriately sized cuff. All patients rested for 5 minutes before BP measurement and were prohibited from smoking and ingesting caffeine for 30 minutes before the measurements. Three measurements were performed at 1-minute intervals, and the average of the last two measurements was taken as the OBP. The 24-hour ABPM was performed using the Oscar 2 (SunTech Medical, Morrisville, NC, USA) and MobileO-Graph (I.E.M. GmbH, Stolberg, Germany) on a typical workday. Daytime was defined as $8 \mathrm{AM}$ to $10 \mathrm{PM}$ and nighttime as $10 \mathrm{PM}$ to $8 \mathrm{AM}$. More than 16 acceptable daytime readings and 12 acceptable nighttime readings were obtained. The time interval between BP measurements was 30 minutes. BP groups were divided according to the 2017 ACC/AHA guidelines. Extreme dippers were defined as patients with a nighttime-daytime systolic BP ratio of $\leq 0.8$; dippers, 0.8 to 0.9 ; non-dippers, 0.9 to 1.0 ; and reverse dippers, $>1.0$.

\section{Statistical analysis}

Statistical analysis was performed using IBM SPSS Statistics 22.0 (IBM Corp., Armonk, NY, USA). Quantitative variables are presented as median with the full range of values, and categorical variables as percentages. Among patients divided into two groups (those who developed ESRD or died and those who did not), Student's $t$ test was used to compare continuous variables and chi-square test or Fisher's exact test was applied to compare categorical variables according to the number of variables. Cox proportional hazard models were used with adjustments for covariates to determine the relationship of BP to the composite outcome. Covariates were selected using correlation analysis with $P<0.05$ and clinical assessment by investigators. Continuous variables of $\mathrm{BP}$ were mean $\mathrm{ABP}$, daytime $A B P$, nighttime $A B P$, and OBP in the systolic and diastolic phases. BP values were also analyzed as categorical variables divided into groups. Mean ABP values were analyzed as three groups: < 125/75 mmHg, between 125/75 and 130/80 $\mathrm{mmHg}$, and > 130/80 mmHg. Daytime ABPs were divided into three groups: $<130 / 80 \mathrm{mmHg}$, between 130/80 and 135/85 $\mathrm{mmHg}$, and > 135/85 mmHg; nighttime ABP values were divided into three groups: $<110 / 65$ $\mathrm{mmHg}$, between 110/65 and 120/70 $\mathrm{mmHg}$, and > 140/85 $\mathrm{mmHg}$.

\section{Results}

\section{Baseline characteristics}

A total of 433 patients agreed to undergo ABPM, and 46 patients were excluded because of patient withdrawal or inappropriate measurements. A total 387 patients were included in this study. The median follow-up period was 40.6 months (range, 2.0-52.5 months). The median age was 61 years, and $58.4 \%$ of patients were men (Table 1 ; Supplementary Table 1, available online). CKD was diagnosed in all patients about 48 months before enrolment. CKD stages were from stage 2 to stage 4 . The median serum creatinine level was $1.6 \mathrm{mg} / \mathrm{dL}$, and the median eGFR calculated using the CKD-EPI equation was 41.7 $\mathrm{mL} / \mathrm{min} / 1.73 \mathrm{~m}^{2}$. The UPCR was $0.7 \mathrm{~g} / \mathrm{g}$ creatinine. Among the 387 patients, normal BP, persistent hyperten- 
Table 1. Clinical characteristics, number and components of antihypertensive therapy, and patterns of blood pressure in participants according to the composite outcome

\begin{tabular}{|c|c|c|c|c|}
\hline Characteristic & $\begin{array}{c}\text { Total } \\
(n=387)\end{array}$ & $\begin{array}{l}\text { ESRD or death } \\
\quad(n=66)\end{array}$ & $\begin{array}{l}\text { Non-ESRD or non-death } \\
(n=321)\end{array}$ & $P$ value \\
\hline Age (yr) & $61(52-69)$ & $62(52-68)$ & $61(51-69)$ & 0.838 \\
\hline Sex, male & $226(58.4)$ & $44(66.7)$ & $182(56.7)$ & 0.135 \\
\hline Duration of CKD (mo) & $68(17-84)$ & $36(13-66)$ & $48(17-91)$ & 0.155 \\
\hline Diabetes mellitus & 139 (35.9) & $45(68.2)$ & $96(29.9)$ & $<0.001$ \\
\hline Body mass index $\left(\mathrm{kg} / \mathrm{m}^{2}\right)$ & $24.7(22.5-27.1)$ & $24.7(22.6-26.6)$ & $24.8(22.5-27.2)$ & 0.599 \\
\hline Cause of CKD & & & & $<0.001$ \\
\hline Diabetic kidney disease & 139 (35.9) & $45(68.2)$ & $96(29.9)$ & \\
\hline Glomerulonephritis & $104(26.9)$ & $8(12.1)$ & $99(30.8)$ & \\
\hline Hypertensive kidney disease & $74(19.1)$ & $7(10.6)$ & $73(22.7)$ & \\
\hline Cystic kidney disease & $8(2.1)$ & $0(0)$ & $9(2.8)$ & \\
\hline Tubulointerstitial disease & $1(0.3)$ & $0(0)$ & $1(0.3)$ & \\
\hline Others & $47(12.1)$ & $6(9.1)$ & $43(13.4)$ & \\
\hline CKD stage & & & & $<0.001$ \\
\hline Stage 1 & $0(0)$ & $0(0)$ & $0(0)$ & \\
\hline Stage 2 & $95(24.5)$ & $3(4.5)$ & $92(28.7)$ & \\
\hline Stage $3 \mathrm{~A}$ & $79(20.4)$ & $2(3.0)$ & $77(24.0)$ & \\
\hline Stage 3B & $93(24.0)$ & $11(16.7)$ & $82(25.5)$ & \\
\hline Stage 4 & $120(31.0)$ & $50(75.8)$ & $70(21.8)$ & \\
\hline Stage 5 & $0(0)$ & $0(0)$ & $0(0)$ & \\
\hline Number of hypertension medications & $2(1-3)$ & $2(2-3)$ & $2(1-3)$ & $<0.001$ \\
\hline Use of ACEi/ARB & $294(76.0)$ & $43(65.2)$ & $251(78.2)$ & 0.024 \\
\hline Use of BB & $123(31.8)$ & $29(43.9)$ & $94(29.3)$ & 0.020 \\
\hline Use of CCB & $240(62.0)$ & $47(71.2)$ & $193(60.1)$ & 0.091 \\
\hline Use of diuretics & $84(21.7)$ & $27(40.9)$ & $57(17.8)$ & $<0.001$ \\
\hline Serum albumin $(g / d L)$ & $4.2(4.0-4.4)$ & $3.9(3.5-4.2)$ & $4.2(4.0-4.4)$ & $<0.001$ \\
\hline Serum BUN (mg/dL) & $24(18-33)$ & $37(32-44)$ & $23(17-28)$ & $<0.001$ \\
\hline Serum $\mathrm{Cr}(\mathrm{mg} / \mathrm{dL})$ & $1.6(1.2-2.2)$ & $2.6(2.0-3.2)$ & $1.5(1.1-1.9)$ & $<0.001$ \\
\hline Serum GFR $\left(\mathrm{mL} / \mathrm{min} / 1.73 \mathrm{~m}^{2}\right)$ & $41.7(28.0-62.1)$ & $22.7(18.8-31.5)$ & $47.0(33.5-65.5)$ & $<0.001$ \\
\hline Serum $\mathrm{Hb}(\mathrm{g} / \mathrm{dL})$ & $12.8(11.3-14.3)$ & $11.4(10.1-12.6)$ & $13.1(11.6-14.5)$ & $<0.001$ \\
\hline Serum HbA1c (\%) & $6.6(5.8-7.5)$ & $7.4(6.6-8.2)$ & $6.4(5.7-7.2)$ & $<0.001$ \\
\hline Serum total-C (mg/dL) & $163(141-191)$ & $159(139-183)$ & $164(141-192)$ & 0.576 \\
\hline Serum Ca $\times \mathrm{P}\left(\mathrm{mg}^{2} / \mathrm{dL}^{2}\right)$ & $32.8(29.1-36.3)$ & $34.0(29.6-39.7)$ & $32.8(28.8-35.8)$ & 0.021 \\
\hline Serum TG (mg/dL) & $133(98-180)$ & $142(92-203)$ & $132(98-178)$ & 0.201 \\
\hline Urine protein-creatinine ratio ( $\mathrm{g} / \mathrm{g}$ creatinine) & $0.7(0.3-1.8)$ & $2.8(1.0-5.4)$ & $0.6(0.2-1.4)$ & $<0.001$ \\
\hline BP pattern & & & & 0.010 \\
\hline Normotensive & $35(9.0)$ & $2(3.0)$ & $33(10.3)$ & \\
\hline Persistent hypertension & $249(64.3)$ & $52(78.8)$ & $197(61.4)$ & \\
\hline Whitecoat hypertension & $33(8.5)$ & $2(3.0)$ & $31(9.7)$ & \\
\hline Masked hypertension & $70(18.1)$ & $10(15.2)$ & $60(18.7)$ & \\
\hline Daytime-nighttime BP ratio & & & & 0.621 \\
\hline Extreme dipper or dipper & $169(43.7)$ & $27(40.9)$ & $142(44.2)$ & \\
\hline Non-dipper or reverse dipper & $218(56.3)$ & $39(59.1)$ & $172(53.6)$ & \\
\hline
\end{tabular}

Data are presented as number (\%) or median (range).

ACEi, angiotensin-converting enzyme inhibitor; ARB, angiotensin receptor blocker; BB, beta blocker; BP, blood pressure; BUN, blood urea nitrogen; Ca $\times$ P, product of calcium and phosphorous; $\mathrm{CCB}$, calcium channel blocker; $\mathrm{CKD}$, chronic kidney disease; Cr, creatinine; ESRD, end-stage renal disease; GFR, glomerular filtration rate; $\mathrm{Hb}$, hemoglobin; HbA1c, hemoglobin A1c; TG, triglycerides; total-C, total cholesterol. 
Table 2. Distribution of blood pressures according to the composite outcome

\begin{tabular}{lrcrr}
\hline & Total & ESRD or death & Non-ESRD or non-death & $P$ value \\
\hline Mean ASBP & $129(94-207)$ & $142(112-206)$ & $128(94-187)$ & $<.001$ \\
Mean ADBP & $79(49-114)$ & $82(60-108)$ & $79(51-114)$ & 0.014 \\
Daytime ASBP & $133(94-213)$ & $146(110-208)$ & $81(52-115)$ & 0.001 \\
Daytime ADBP & $82(52-115)$ & $84(64-113)$ & $118(87-181)$ & 0.025 \\
Nighttime ASBP & $121(87-197)$ & $131(110-197)$ & $72(44-117)$ & 0.001 \\
Nighttime ADBP & $73(42-117)$ & $77(53-112)$ & $132(90-207)$ & 0.020 \\
OSBP & $133(90-207)$ & $140(100-205)$ & $79(40-115)$ & 0.048 \\
ODBP & $78(30-115)$ & $75(30-104)$ & & 0.785 \\
\hline
\end{tabular}

Data for blood pressure are presented as median (range).

ADBP, ambulatory diastolic blood pressure; ASBP, ambulatory systolic blood pressure; ESRD, end-stage renal disease; ODBP, office diastolic blood pressure; OSBP, office systolic blood pressure.

sion, whitecoat hypertension, and masked hypertension were observed in 35 (9.0\%), 249 (64.3\%), 33 (8.5\%), and 70 (18.1\%) patients, respectively. A total of 218 patients (56.3\%) were non-dippers or reverse dippers. A total of 139 patients (35.9\%) were diagnosed with DM. Etiologies of CKD were DM, glomerulonephritis, hypertension, cystic kidney disease, and tubulointerstitial disease, which were observed in 139 patients (35.9\%), 104 patients (26.9\%), 74 patients (19.1\%), 8 patients $(2.1 \%)$, and 1 patient $(0.3 \%)$, respectively.

The median serum creatinine level was $2.7 \mathrm{mg} / \mathrm{dL}$ in the ESRD or death group and $1.6 \mathrm{mg} / \mathrm{dL}$ in the non-ESRD or non-death group $(P<0.001)$. The median serum GFR was $22.7 \mathrm{~mL} / \mathrm{min} / 1.73 \mathrm{~m}^{2}$ in the ESRD or death group and $47.0 \mathrm{~mL} / \mathrm{min} / 1.73 \mathrm{~m}^{2}$ in the non-ESRD or non-death group $(P<0.001)$. CKD stage $2,3 \mathrm{~A}, 3 \mathrm{~B}$, and 4 was observed in 3 (4.5\%), 2 (3.0\%), 11 (16.7\%), and 50 (75.8\%) patients in the ESRD or death group, respectively, and in $92(28.7 \%), 77(24.0 \%), 82(25.5 \%)$, and 70 (21.8\%) patients in the non-ESRD or non-death group, respectively. The UPCR ratio was 2.8 in the ESRD or death group and 0.6 in the non-ESRD or non-death group. The incidence of whitecoat hypertension was $3.0 \%$ in the ESRD or death group and $9.7 \%$ in the non-ESRD or non-death group. The incidence of masked hypertension was $15.2 \%$ in the ESRD or death group and $18.7 \%$ in the non-ESRD or nondeath group. The distribution of daytime-nighttime BP ratio was not different between the ESRD or death group and the non-ESRD or non-death group. The number of hypertension medications was a median of 2 in the ESRD or death group, which was higher than that in the nonESRD or non-death group. The number of patients with DM was $45(68.2 \%)$ in the ESRD or death group and 96
(29.9\%) in the non-ESRD or non-death group. The median glycated hemoglobin value was $6.6 \%$ in the total patient group, and $7.4 \%$ and $6.4 \%$ in in the ESRD or death group and the non-ESRD or non-death group, respectively $(P<0.001)$.

\section{Distribution of $A B P$ and $O B P$}

The mean ABP was $129 / 79 \mathrm{mmHg}$, and the mean OBP was 133/78 mmHg (Table 2, Supplementary Table 2). In the ESRD or death group, the daytime ambulatory systolic BP (ASBP) was $146 \mathrm{mmHg}$ and the nighttime ASBP was $131 \mathrm{mmHg}$; the median daytime ambulatory diastolic BP (ADBP) was $84 \mathrm{mmHg}$, and the nighttime ADBP was 77 mmHg. The ABP values were higher in the ESRD or death group than in the non-ESRD or non-death group, similar to office systolic BP (OSBP). However, the difference in office diastolic BP (ODBP) between the two groups was not significant $(P=0.785)$. The square of the correlation coefficient $\left(\mathrm{R}^{2}\right)$ between mean ASBP and OSBP was 0.210 and that between mean ADBP and ODBP was 0.228 (Fig. 2, Supplementary Fig. 1). The $\mathrm{R}^{2}$ value was 0.213 in the systolic phase and 0.250 in the diastolic phase between OBP and mean daytime ABP, and 0.168 in the systolic phase and 0.159 in the diastolic phase between OBP and mean nighttime ABP.

\section{Composite outcome and $B P$}

Among the total 387 patients who were analyzed, 66 (17.0\%) cases had the composite outcome (ESRD or death). One patient who developed ESRD and died in the follow-up period was analyzed as having ESRD. There 
A

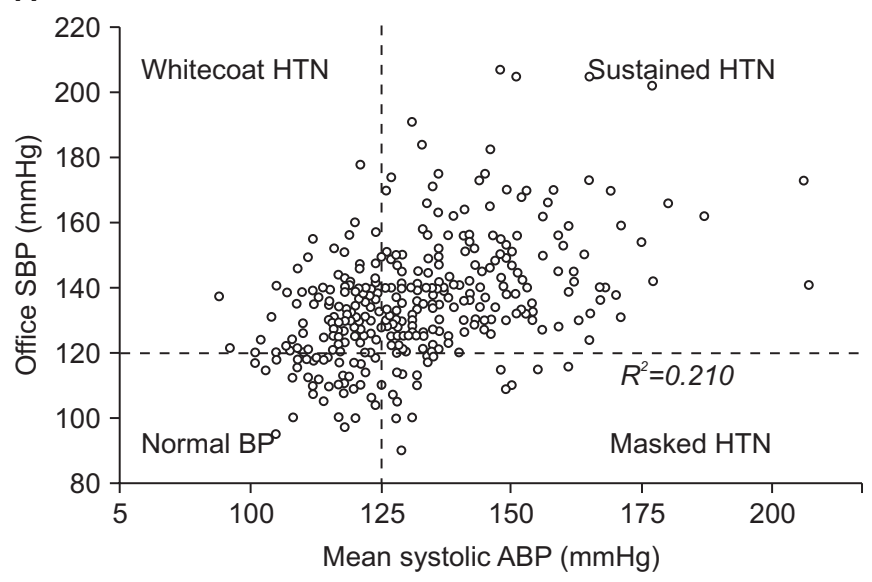

B

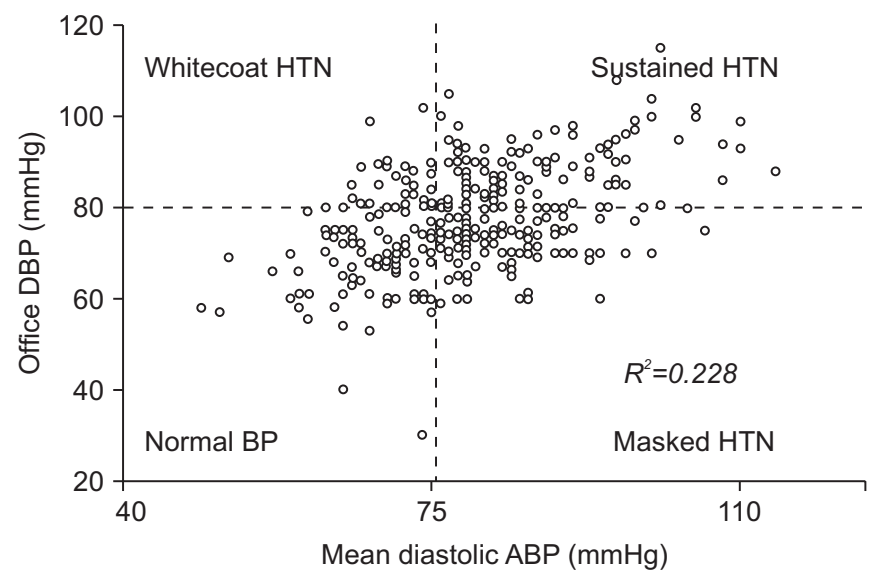

Figure 2. Relationships between office blood pressure (BP) and mean ambulatory blood pressure in the (A) systolic and (B) diastolic phase.

ABP, ambulatory BP; DBP, diastolic BP; HTN, hypertension; SBP, systolic BP.

were two foreign patients whose death could not be confirmed; however, as they developed ESRD in the followup period, they were classified as having the composite outcome.

The mean ASBP and ADBP increased the risk of ESRD or death, with adjusted hazard ratios (HRs) of 1.03 (95\% confidence interval [CI], 1.01-1.04; $P<0.001)$ and 1.04 (95\% CI, 1.02-1.07; $P=0.002$ ), respectively (Table 3 , Supplementary Table 3). Higher daytime and nighttime ABPs in the systolic and diastolic phases were also risk factors for ESRD or death (daytime ASBP: HR, 1.03; 95\% CI, 1.01-1.04; $P<0.001$; daytime ADBP: HR, 1.04; 95\% CI, 1.02-1.07; $P=0.001$; nighttime ASBP: HR, 1.02; 95\% CI, 1.01-1.04; $P=0.001$; nighttime ADBP: HR, 1.04; 95\% CI, 1.01-1.06; $P=0.003$ ), whereas OSBP and ODBP were not statistically significant. When the outcome was ESRD only, daytime ASBP, daytime ADBP, nighttime ASBP, and nighttime ADBP increased the risk of ESRD by 1.03 (95\% CI, 1.01-1.04; $P<0.001), 1.05$ (95\% CI, 1.02-1.07; $P=$ 0.001 ), 1.02 (95\% CI, 1.00-1.03; $P=0.013$ ), and $1.03(95 \%$ CI, 1.00-1.05; $P=0.021$ ), respectively.

The ABP groups were related to an increased risk of ESRD or death, whereas the OBP groups were not (Fig. 3). Patients with CKD with mean ABP between 125/75 and $130 / 80 \mathrm{mmHg}$ showed a statistically insignificant difference in the rate of ESRD or death compared to those with mean ABP $<125 / 75 \mathrm{mmHg}(P=0.147)$. The HR of patients with mean $\mathrm{ABP}>130 / 80 \mathrm{mmHg}$ was $4.79(95 \%$ CI, 1.68-13.70; $P=0.003$ ). When the outcome was ESRD only, the HR of $\mathrm{BP}>130 / 80 \mathrm{mmHg}$ by mean $\mathrm{ABP}$ was 7.57
(95\% CI, 1.80-32.57; $P=0.006$ ) and that of BP between 125/75 and 130/80 mmHg was 8.761 (95\% CI, 1.75-43.96; $P=0.008)$ compared with $\mathrm{ABP}<125 / 75 \mathrm{mmHg}$.

Daytime ABP > 135/85 mmHg increased the risk of ESRD or death by 3.54 -fold compared with daytime ABP $<130 / 80$ mmHg. Daytime ABP between 130/80 and $135 / 85 \mathrm{mmHg}$ also showed a 2.45 -fold higher risk. When the outcome was ESRD only, however, there were no differences among the daytime ABP groups. The HR of ESRD or death increased when each daytime ASBP or ADBP group became higher. There were no patients with ESRD or mortality in the normal nighttime ABP group.

\section{Subgroup analysis}

The HR of mean ASBP was 1.04 in elderly patients ( $\geq 65$ years) (95\% CI, 1.02-1.06; $P<0.001$ ) (Table 4$)$. The risk of ESRD or death significantly increased in women (HR, 1.04; 95\% CI, 1.02-1.06; $P<0.001)$. Patients with CKD and DM showed a 1.02-fold higher risk of ESRD or death, and those without DM showed a 1.05-fold higher risk of ESRD or death when the mean ASBP increased by $1 \mathrm{mmHg}$. The CKD stage 4 subgroup showed a 1.03-fold increased risk when the mean ASBP became higher (HR, 1.03; 95\% CI, 1.01-1.05; $P=0.001$ ). In patients with proteinuria (UPCR $\geq$ $0.5 \mathrm{~g} / \mathrm{g}$ creatinine), there was a higher risk of ESRD or death as the mean ASBP became higher (HR, 1.03; 95\% CI, 1.011.05; $P=0.002)$. In non-dippers or reverse dippers, higher ASBP increased the risk of ESRD or death (HR for ESRD or death, 1.03; 95\% CI, 1.01-1.04; $P=0.002$; HR for ESRD only, 
Table 3. Adjusted hazard ratios of each component of ABPM and OBP in patients with CKD

\begin{tabular}{|c|c|c|c|c|c|c|}
\hline & \multicolumn{3}{|c|}{ Model 1: ESRD or death ${ }^{b}$} & \multicolumn{3}{|c|}{ Model 2: ESRD only ${ }^{\mathrm{C}}$} \\
\hline & HR & $95 \% \mathrm{Cl}$ & $P$ value & HR & $95 \% \mathrm{Cl}$ & $P$ value \\
\hline ABP group (mmHg) & & & 0.001 & & & $<0.001$ \\
\hline$<125 / 75$ & & Ref. & & & Ref. & \\
\hline $125 / 75-130 / 80$ & 2.56 & $0.72-9.12$ & 0.147 & 8.76 & $1.75-43.96$ & 0.008 \\
\hline$\geq 130 / 80$ & 4.79 & $1.68-13.70$ & 0.003 & 7.65 & $1.80-32.57$ & 0.006 \\
\hline Mean ASBP & 1.03 & $1.01-1.04$ & $<0.001$ & 1.03 & $1.01-1.04$ & 0.002 \\
\hline Mean ASBP (group) (mmHg) & & & 0.002 & & & 0.002 \\
\hline$<125$ & & Ref. & & & Ref. & \\
\hline $125-130$ & 2.75 & $0.84-8.95$ & 0.094 & 4.73 & $1.35-16.63$ & 0.418 \\
\hline$\geq 130$ & 3.72 & $1.62-8.55$ & 0.002 & 4.07 & $1.63-10.13$ & 0.040 \\
\hline Mean ADBP & 1.04 & $1.02-1.07$ & 0.002 & 1.05 & $1.02-1.08$ & 0.001 \\
\hline Mean ADBP (group) (mmHg) & & & $<0.001$ & & & $<0.001$ \\
\hline$<75$ & & Ref. & & & Ref. & \\
\hline $75-80$ & 1.05 & $0.45-2.44$ & 0.917 & 1.35 & $0.51-3.58$ & 0.546 \\
\hline$>80$ & 2.59 & $1.39-4.87$ & 0.003 & 2.93 & $1.42-6.03$ & 0.004 \\
\hline Daytime ABP (group) (mmHg) & & & 0.001 & & & 0.131 \\
\hline$<130 / 80$ & & Ref. & & & & \\
\hline $130 / 80-135 / 85$ & 2.45 & $0.85-7.04$ & 0.096 & & - & \\
\hline$\geq 135 / 85$ & 3.54 & $1.64-7.65$ & 0.001 & & - & \\
\hline Daytime ASBP & 1.03 & $1.01-1.04$ & $<0.001$ & 1.03 & $1.01-1.04$ & $<0.001$ \\
\hline Daytime ASBP (group) (mmHg) & & & 0.002 & & & 0.142 \\
\hline$<130$ & & Ref. & & & & \\
\hline $130-135$ & 2.27 & $0.85-7.04$ & 0.102 & & - & \\
\hline$\geq 135$ & 3.07 & $1.55-6.06$ & 0.001 & & - & \\
\hline Daytime ADBP & 1.04 & $1.02-1.07$ & 0.001 & 1.05 & $1.02-1.07$ & 0.001 \\
\hline Daytime ADBP (group) (mmHg) & & & 0.009 & & & 0.004 \\
\hline$<80$ & & Ref. & & & Ref. & \\
\hline $80-85$ & 1.12 & $0.51-2.49$ & 0.778 & 1.40 & $0.59-3.35$ & 0.450 \\
\hline$\geq 85$ & 2.39 & $1.32-4.33$ & 0.004 & 2.89 & $1.50-5.56$ & 0.032 \\
\hline OSBP & & & 0.834 & & & 0.780 \\
\hline ODBP & & & 0.742 & & & 0.176 \\
\hline
\end{tabular}

ABP, ambulatory blood pressure; ABPM, ABP monitoring; ADBP, ambulatory diastolic blood pressure; ASBP, ambulatory systolic blood pressure; $\mathrm{Cl}$, confidence interval; CKD, chronic kidney disease; ESRD, end-stage renal disease; HR, hazard ratio; OBP, office blood pressure; ODBP, office diastolic blood pressure; OSBP, office systolic blood pressure; Ref., references.

${ }^{a}$ Groups of office blood pressure could not be included in these Cox regression models. ${ }^{b}$ Model 1 was adjusted for age; sex; diabetes mellitus; number of hypertension medications; use of angiotensin-converting enzyme inhibitor (ACEi) or angiotensin II receptor blocker (ARB), use of beta blocker (BB); serum level of blood urea nitrogen (BUN); estimated glomerular filtration rate (eGFR) calculated using the Chronic Kidney Disease Epidemiology Collaboration (CKD-EPI) equation; albumin; sodium; high-density lipoprotein; and urine protein-creatinine ratio (UPCR). 'Model 2 was adjusted for age; sex; diabetes mellitus; number of hypertension medications; use of ACEi or ARB, BB, diuretics, and alpha blocker; serum level of BUN; eGFR calculated using the CKD-EPI equation; albumin; intact parathyroid hormone; hemoglobin; total bicarbonate; chloride; protein; hematocrit; alkaline phosphatase; product of calcium and phosphorous; sodium and potassium; and UPCR.

1.03; 95\% CI, 1.01-1.05; $P=0.004)$.

\section{Discussion}

By comparing $\mathrm{ABP}$ and $\mathrm{OBP}$, this study showed the importance of $\mathrm{ABP}$ in predicting the prognosis of patients with CKD with respect to ESRD or death. The risks of ESRD or death became higher as the ABP values (mean, daytime, and nighttime) increased; however, this was not observed for OBP values. Among the components of ABP, mean ASBP had the strongest correlation with the composite outcome (ESRD or death). 
A

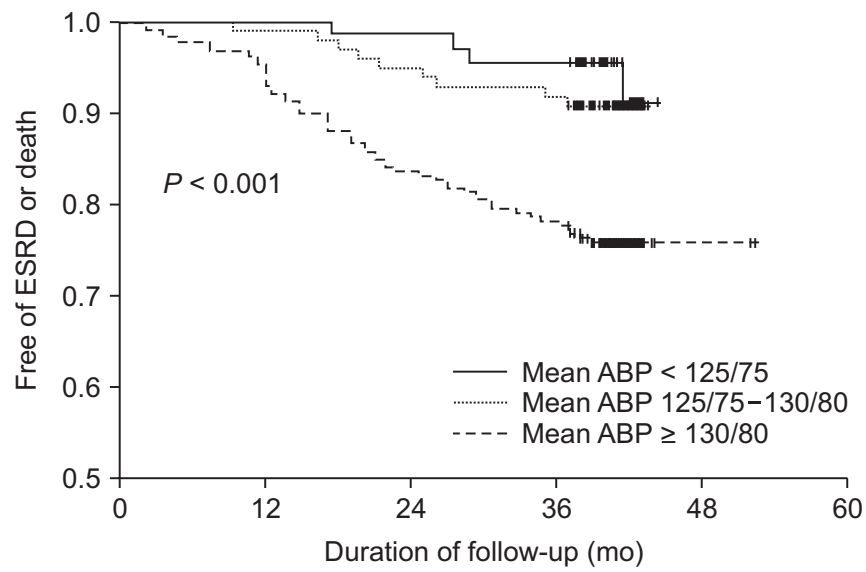

B

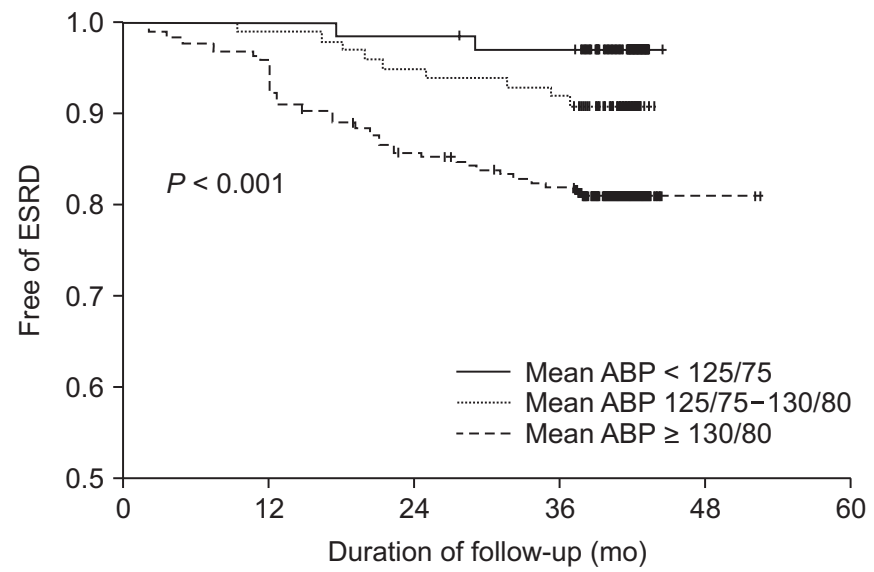

Figure 3. The proportion of patients free from the composite outcome (A) or risk of end-stage renal disease (ESRD) (B) stratified by mean ambulatory blood pressures (ABPs).

Table 4. Adjusted hazard ratios with increasing ASBP by $1 \mathrm{mmHg}$ in subgroups

\begin{tabular}{|c|c|c|c|c|c|c|}
\hline & \multicolumn{3}{|c|}{ Model 1: ESRD or death ${ }^{a}$} & \multicolumn{3}{|c|}{ Model 2: ESRD only ${ }^{\mathrm{b}}$} \\
\hline & HR & $95 \% \mathrm{Cl}$ & $P$ value & HR & $95 \% \mathrm{Cl}$ & $P$ value \\
\hline \multicolumn{7}{|l|}{ Age (yr) } \\
\hline$\geq 65$ & 1.04 & $1.02-1.06$ & $<0.001$ & & uc & 0.051 \\
\hline$<65$ & & uc & 0.295 & & uc & 0.065 \\
\hline \multicolumn{7}{|l|}{ Sex } \\
\hline Female & 1.04 & $1.02-1.06$ & $<0.001$ & 1.04 & $1.01-1.06$ & 0.002 \\
\hline Male & & uc & 0.353 & & uc & 0.192 \\
\hline \multicolumn{7}{|l|}{ UPCR (g/g creatinine) } \\
\hline$\geq 0.5$ & 1.03 & $1.01-1.04$ & 0.002 & 1.03 & $1.01-1.04$ & 0.003 \\
\hline$<0.5$ & & uc & 0.547 & & uc & 0.594 \\
\hline \multicolumn{7}{|l|}{ Nighttime-daytime ASBP ratio } \\
\hline Non-dipper or reverse dipper & 1.03 & $1.01-1.04$ & 0.002 & 1.03 & $1.01-1.05$ & 0.004 \\
\hline Dipper & & uc & 0.105 & & uc & 0.071 \\
\hline \multicolumn{7}{|l|}{$\mathrm{DM}$} \\
\hline DM & 1.02 & $1.00-1.04$ & 0.036 & & uc & 0.250 \\
\hline Non-DM & 1.05 & $1.02-1.08$ & 0.003 & & uc & 0.560 \\
\hline
\end{tabular}

ASBP, ambulatory systolic blood pressure; Cl, confidence interval; DM, diabetes mellitus; ESRD, end-stage renal disease; HR, hazard ratio; uc, unable to calculate; UPCR, urine protein-creatinine ratio.

${ }^{a}$ Model 1 was adjusted for age; sex; diabetes mellitus; number of hypertension medications; use of angiotensin-converting enzyme inhibitor (ACEi) or angiotensin II receptor blocker (ARB), use of beta blocker (BB); serum level of blood urea nitrogen (BUN); estimated glomerular filtration rate (eGFR) calculated using the Chronic Kidney Disease Epidemiology Collaboration (CKD-EPI) equation; albumin; sodium; high-density lipoprotein; and urine protein-creatinine ratio (UPCR). ${ }^{b}$ Model 2 was adjusted for age; sex; diabetes mellitus; number of hypertension medications; use of ACEi or ARB, BB, diuretics, and alpha blocker; serum level of BUN; eGFR calculated using the CKD-EPI equation; albumin; intact parathyroid hormone; hemoglobin; total bicarbonate; chloride; protein; hematocrit; alkaline phosphatase; product of calcium and phosphorous; sodium and potassium; and UPCR.

This is the first study to report that ABP is a more accurate prognostic marker than OBP in predicting severe renal function deterioration or mortality in patients with CKD in Korea. In previous studies that compared ABP and conventional OBP in the general population, $\mathrm{ABP}$ was suggested to have good prognostic value for cardiovascular events or mortality rate [13-16]. For patients with CKD showing BP variability, ABP has significance for not only cardiovascular outcomes but also renal outcomes $[17,18]$. In this study, after adjusting for various 
possible confounding factors, we concluded that $\mathrm{ABP}$ itself is a reliable measurement.

Controversy remains regarding the appropriate time of BP measurement in relation to renal outcomes or death. In the present study, daytime BP was of greater importance in predicting ESRD, whereas nighttime BP was of greater importance $[19,20]$. In a study on 682 hypertensive patients who underwent echocardiography, nocturnal BP, rather than non-dipping, showed better prediction of left ventricular hypertrophy (LVH), especially in patients with a high cardiovascular risk [21]. We observed that the groups of mean $\mathrm{ABP}$ had a greater correlation with the composite outcome or ESRD only than those of daytime ABP or OBP. Repeated measurements of $\mathrm{BP}$ may explain the better predictive power of mean $\mathrm{ABP}$. However, as taking 24-hour monitoring device each time could cause discomfort, an appropriate timing of onetime BP measurement that can replace ABP is desired, and further studies are needed in this regard [22].

Many studies have shown that cardiovascular risk and mortality rate are improved by intensive BP lowering [23]. These studies did not show better outcomes in patients with CKD, except for one study [7]. There has been a criticism that the BP measurement method in the SPRINT study was equivalent to self-monitored BP measurement. In our study, among patients divided according to the correlated ABP stages in the 2017 ACC/AHA guidelines, those with $A B P>125 / 75 \mathrm{mmHg}$ showed a higher risk of ESRD or death than those with ABP $<125 / 75 \mathrm{mmHg}$, which is the current normal ABP criteria. However, the model of OBP stages had no statistical importance in explaining the risk of ESRD or death. This suggests the availability of an $\mathrm{ABP}$ target for predicting CKD progression.

In subgroup analysis, the non-dipping pattern of BP was correlated with the outcome of ESRD or death. Over $50 \%$ of the current study group were non-dippers and reverse dippers. The results showed that ABP more accurately indicates the prognosis of patients with $\mathrm{CKD}$ with a non-dipping pattern of BP than that of patients with a dipping pattern, which is consistent with the result of a previous study [24]. Another study that analyzed 1,317 hypertensive patients with CKD stages 2 to 4 revealed specific clinical characteristics among the dipping patterns, and patients in the non-dipping/reverse dipping group showed greater renal dysfunction and extent of proteinuria [25]. Previous studies have shown that hypertension, especially a high night BP or a reverse dipping pattern, causes pressure overload leading to LVH, which results in end-organ damage related to poor cardiovascular outcomes [26-28]. As LVH is also a strong risk factor of mortality or renal function deterioration in patients with CKD [29-31], the pattern of BP has been suggested as a strong predictor.

This study has some limitations. First, a longer followup period is needed to explain the prognostic value of nighttime BP. During the follow-up period, there was no patient who developed ESRD or died in the normotensive group when measuring nighttime ABP. Therefore, we could not suggest that lowering nighttime BP would be appropriate for patients with early-stage CKD. Second, according to the European Society of Hypertension guidelines [32], accurate measurement of $A B P$ requires an appropriate device, software, patient education, and editing data, which are barriers to its clinical application.

In conclusion, ABP showed better associations with renal outcome and death than OBP. The results of the present study suggest that ABP could be a prognostic factor for renal outcome and death in patients with CKD. To clarify the optimal BP target using ABP, further prospective studies or studies on cost-effectiveness should be conducted in patients with CKD.

\section{Conflicts of interest}

All authors have no conflicts of interest to declare.

\section{Authors' contributions}

Hyung Eun Son, Ho Jun Chin, Yoon Kyu Oh, and KookHwan Oh participated in design of the study. Hyung Eun Son, Kipyo Kim, and Youngjin Yi participated in analysis of data. Hyung Eun Son, Ji Young Ryu, and Suryeong Go participated in collection of data and Hyung Eun Son was in charge of writing the paper.

\section{References}

[1] Park JI, Baek H, Jung HH. Prevalence of chronic kidney disease in Korea: the Korean National Health and Nutritional Examination Survey 2011-2013. J Korean Med Sci 2016;31: 915-923. 
[2] Whelton PK, Carey RM, Aronow WS, et al. 2017 ACC/AHA/ AAPA/ABC/ACPM/AGS/APhA/ASH/ASPC/NMA/PCNA guideline for the prevention, detection, evaluation, and management of high blood pressure in adults: a report of the American College of Cardiology/American Heart Association Task Force on clinical practice guidelines. J Am Coll Cardiol 2018;71:e127-e248.

[3] Judd E, Calhoun DA. Management of hypertension in CKD: beyond the guidelines. Adv Chronic Kidney Dis 2015;22: 116-122.

[4] Appel LJ, Wright JT Jr, Greene T, et al.; AASK Collaborative Research Group. Intensive blood-pressure control in hypertensive chronic kidney disease. N Engl J Med 2010;363:918929.

[5] Klahr S, Levey AS, Beck GJ, et al. The effects of dietary protein restriction and blood-pressure control on the progression of chronic renal disease. Modification of Diet in Renal Disease Study Group. N Engl J Med 1994;330:877-884.

[6] Ruggenenti P, Perna A, Loriga G, et al.; REIN-2 Study Group. Blood-pressure control for renoprotection in patients with non-diabetic chronic renal disease (REIN-2): multicentre, randomised controlled trial. Lancet 2005;365:939-946.

[7] Cheung AK, Rahman M, Reboussin DM, et al.; SPRINT Research Group. Effects of intensive BP control in CKD. J Am Soc Nephrol 2017;28:2812-2823.

[8] Pogue V, Rahman M, Lipkowitz M, et al.; African American Study of Kidney Disease and Hypertension Collaborative Research Group. Disparate estimates of hypertension control from ambulatory and clinic blood pressure measurements in hypertensive kidney disease. Hypertension 2009; 53:20-27.

[9] Gorostidi M, Sarafidis PA, de la Sierra A, et al.; Spanish ABPM Registry Investigators. Differences between office and 24-hour blood pressure control in hypertensive patients with CKD: a 5,693-patient cross-sectional analysis from Spain. Am J Kidney Dis 2013;62:285-294.

[10] Gustavsen PH, Høegholm A, Bang LE, Kristensen KS. White coat hypertension is a cardiovascular risk factor: a 10-year follow-up study. J Hum Hypertens 2003;17:811-817.

[11] Ohkubo T, Kikuya M, Metoki H, et al. Prognosis of "masked" hypertension and "white-coat" hypertension detected by 24-h ambulatory blood pressure monitoring 10-year followup from the Ohasama study. J Am Coll Cardiol 2005;46:508515.

[12] Oh YK, Chin HJ, Ahn SY, et al. Discrepancies in clinic and ambulatory blood pressure in Korean chronic kidney dis- ease patients. J Korean Med Sci 2017;32:772-781.

[13] Verdecchia P, Porcellati C, Schillaci G, et al. Ambulatory blood pressure. An independent predictor of prognosis in essential hypertension. Hypertension 1994;24:793-801.

[14] Ohkubo T, Imai Y, Tsuji I, et al. Reference values for 24hour ambulatory blood pressure monitoring based on a prognostic criterion: the Ohasama Study. Hypertension 1998;32:255-259.

[15] Staessen JA, Thijs L, Fagard R, et al. Predicting cardiovascular risk using conventional vs ambulatory blood pressure in older patients with systolic hypertension. Systolic Hypertension in Europe Trial Investigators. JAMA 1999;282:539546.

[16] Sega R, Facchetti R, Bombelli M, et al. Prognostic value of ambulatory and home blood pressures compared with office blood pressure in the general population: followup results from the Pressioni Arteriose Monitorate e Loro Associazioni (PAMELA) study. Circulation 2005;111:17771783.

[17] Agarwal R, Andersen MJ. Blood pressure recordings within and outside the clinic and cardiovascular events in chronic kidney disease. Am J Nephrol 2006;26:503-510.

[18] Minutolo R, Agarwal R, Borrelli S, et al. Prognostic role of ambulatory blood pressure measurement in patients with nondialysis chronic kidney disease. Arch Intern Med 2011; 171:1090-1098.

[19] Agarwal R, Andersen MJ. Prognostic importance of ambulatory blood pressure recordings in patients with chronic kidney disease. Kidney Int 2006;69:1175-1180.

[20] Katafuchi E, Nakayama M, Tanaka S, et al. Comparison of prognostic values of daytime and night-time systolic blood pressures on renal outcomes in patients with chronic kidney disease. Circ J 2017;81:1454-1462.

[21] Yi JE, Shin J, Ihm SH, et al. Not nondipping but nocturnal blood pressure predicts left ventricular hypertrophy in the essential hypertensive patients: the Korean ambulatory blood pressure multicenter observational study. J Hypertens 2014;32:1999-2004.

[22] Ryu J, Cha RH, Kim DK, et al. Time points for obtaining representative values of 24-hour blood pressure in chronic kidney disease. Korean J Intern Med 2015;30:665-674.

[23] Xie X, Atkins E, Lv J, et al. Effects of intensive blood pressure lowering on cardiovascular and renal outcomes: updated systematic review and meta-analysis. Lancet 2016;387:435443.

[24] Banegas JR, Ruilope LM, de la Sierra A, et al. Relationship 
between clinic and ambulatory blood-pressure measurements and mortality. N Engl J Med 2018;378:1509-1520.

[25] Cha RH, Kim S, Ae Yoon S, et al. Association between blood pressure and target organ damage in patients with chronic kidney disease and hypertension: results of the APrODiTe study. Hypertens Res 2014;37:172-178.

[26] Ben-Dov IZ, Kark JD, Ben-Ishay D, Mekler J, Ben-Arie L, Bursztyn M. Predictors of all-cause mortality in clinical ambulatory monitoring: unique aspects of blood pressure during sleep. Hypertension 2007;49:1235-1241.

[27] Kim BK, Kim YM, Lee Y, Lim YH, Shin J. A reverse dipping pattern predicts cardiovascular mortality in a clinical cohort. J Korean Med Sci 2013;28:1468-1473.

[28] Fagard RH, Thijs L, Staessen JA, Clement DL, De Buyzere ML, De Bacquer DA. Night-day blood pressure ratio and dipping pattern as predictors of death and cardiovascular events in hypertension. J Hum Hypertens 2009;23:645-653.

[29] Shlipak MG, Fried LF, Cushman M, et al. Cardiovascular mortality risk in chronic kidney disease: comparison of traditional and novel risk factors. JAMA 2005;293:1737-1745.

[30] Middleton RJ, Parfrey PS, Foley RN. Left ventricular hypertrophy in the renal patient. J Am Soc Nephrol 2001;12:10791084.

[31] Leoncini G, Viazzi F, Parodi D, et al. Mild renal dysfunction and subclinical cardiovascular damage in primary hypertension. Hypertension 2003;42:14-18.

[32] O'Brien E, Asmar R, Beilin L, et al.; European Society of Hypertension Working Group on Blood Pressure Monitoring. European Society of Hypertension recommendations for conventional, ambulatory and home blood pressure measurement. J Hypertens 2003;21:821-848. 